\title{
Rheumatological Assessment Is Important for Interstitial Lung Disease Diagnosis
}

\author{
Yair Levi, Lilach Israeli-Shani, Michael Kuchuk, Gali Epstein Shochet ${ }^{\circledR}$, Matthew Koslow, \\ and David Shitrit
}

ABSTRACT. Objective. Interstitial lung diseases (ILD) form a diverse group of parenchymal lung disorders. Currently, a multidisciplinary team (MDT) including pulmonologists, radiologists, and pathologists is the gold standard for ILD diagnosis. Recently, additional subtypes of connective tissue disease (CTD)-ILD with autoimmune features were defined, making the rheumatological assessment increasingly important. We aimed to assess the effect of adding a rheumatologist to the MDT for routine rheumatology assessment.

Methods. A prospective study that assessed newly diagnosed ILD patients by 2 parallel blinded arms; all patients were evaluated by both MDT (e.g., history, physical examination, blood tests, pulmonary function tests, and biopsies, if needed) and a rheumatologist (e.g., history, physical examination, blood and serological tests).

Results. Sixty patients were assessed with the mean age of $67.3 \pm 12$ years, $55 \%$ male, and $28 \%$ smokers. The rheumatological assessment reclassified $21 \%$ of the idiopathic pulmonary fibrosis as CTD. Moreover, the number of CTD-ILD with autoimmune features was increased by $77 \%$. These included antineutrophil cytoplasmic antibody-associated vasculitis, antisynthetase syndrome, and IgG4-related ILD. Retrospectively, rheumatological evaluation could have saved 7 bronchoscopies and 1 surgical biopsy.

Conclusion. Adding routine rheumatology assessments could significantly increase diagnostic accuracy and reduce invasive procedures. (First Release August 15 2018; J Rheumatol 2018; 45:1509-14; doi:10.3899/jrheum.171314)

Key Indexing Terms:
CONNECTIVE TISSUE DISEASES
PULMONARY FIBROSIS

Interstitial lung diseases (ILD) are a group of diffuse parenchymal lung disorders that are classified according to specific clinical, radiological, and histopathological features ${ }^{1}$. Often, the ILD has no identifiable underlying cause, with a usual interstitial pneumonia (UIP) pattern in the high-resolution computed tomography (HRCT) scan, and is regarded as idiopathic pulmonary fibrosis (IPF). Frequently, however, it is associated with a specific environmental exposure or underlying connective tissue disease (CTD).

From the Internal Medicine Department E and Pulmonary Department, Meir Medical Center, Kfar Saba; Sackler Faculty of Medicine, Tel Aviv University, Tel Aviv; Internal Medicine Department A, Hillel Yaffe Medical Center, Hadera, Israel; Pulmonary and Critical Care Medicine, Mayo Clinic, Rochester, Minnesota, USA.

Y. Levi, MD, Internal Medicine Department E, Meir Medical Center, and Sackler Faculty of Medicine, Tel Aviv University; L. Israeli-Shani, MD, Pulmonary Department, Meir Medical Center; M. Kuchuk, MD, Internal Medicine Department A, Hillel Yaffe Medical Center; G. Epstein Shochet, PhD, Pulmonary Department, Meir Medical Center; M. Koslow, MD, Pulmonary and Critical Care Medicine, Mayo Clinic; D. Shitrit, MD, Pulmonary Department, Meir Medical Center, and Sackler Faculty of Medicine, Tel Aviv University. Y. Levi and L. Israeli-Shani contributed equally to this study.

Address correspondence to Prof. D. Shitrit, Pulmonary Department, Meir Medical Center, Tshernichovsky 59, Kfar Saba 4428164, Israel. E-mail:davids3@clalit.org.il

Accepted for publication April 18, 2018.

\section{INTERSTITIAL LUNG DISEASE RHEUMATIC DISEASES}

CTD causes a myriad of pulmonary complications, including bronchiolitis, bronchiectasis, pleuritis, pulmonary hypertension, and ILD, all called CTD-ILD $2,3,4$. A multidisciplinary team (MDT), including a pulmonologist, radiologist, and pathologist, is currently the gold standard for diagnosing $\mathrm{ILD}^{5}$. However, the diagnosis of CTD-ILD is one of the most pressing challenges in our field. Although it is common for the ILD to be diagnosed concurrently or after CTD, some patients present with ILD years prior to the CTD diagnosis. Additionally, some patients may present with autoimmune disease dominated by or limited to pulmonary manifestations ${ }^{6}$. Recently, a new, important subtype of CTD-ILD was defined: interstitial pneumonitis with autoimmune features (IPAF), indicating the importance of a careful, systematic approach to ILD diagnosis.

Therefore, the input of rheumatologists in ILD diagnosis has become increasingly important. We conducted a blinded, prospective study to assess the effect of adding a rheumatologist to the MDT to conduct a rheumatological assessment for ILD diagnosis.

\section{MATERIALS AND METHODS}

Study design. This was a prospective study of patients attending the Pulmonary Clinic at Meir Medical Center, a referral hospital in central Israel

Personal non-commercial use only. The Journal of Rheumatology Copyright $\odot$ 2018. All rights reserved. 
serving over 750,000 people. Patients with a new diagnosis of ILD for the period from June 1, 2015, through June 30, 2016, who met the criteria of age $>18$ years and new-onset ILD based on American Thoracic Society (ATS) criteria $^{7}$, were included. Exclusion criteria were preexisting CTD and other known causes of ILD, including family history, toxic effects of medications, occupational or environmental exposure, aspiration-induced lung disease, and acute interstitial pneumonitis.

All new ILD patients $(n=60)$ underwent routine MDT assessment, followed by a blinded assessment by a team of rheumatologists. Then, the MDT diagnosis was revised following a rheumatologist's input (Figure 1). The diagnosis of IPF was based on the ATS guidelines and included the UIP pattern in an HRCT scan and the absence of identifiable underlying cause or pathological diagnosis in case of biopsy ${ }^{5}$.

Multidisciplinary pulmonology team evaluation. Each patient received a complete standardized pulmonology assessment (Supplementary Data 1, available with the online version of this article), including repeated detailed history, family history of ILD, medications and environmental risk factors, as well as a physical examination; in addition, complete blood count, chemistry, renal and liver function tests, antinuclear antibody, rheumatoid factor (RF), C-reactive protein (CRP), anti-dsDNA, Scl70, anti-SSA, and anti-SSB were done. A cyclic citrullinated peptide (CCP) antibodies test was done in the case of a positive RF result. In addition, all patients underwent HRCT and pulmonary function testing, including spirometry, lung volume test, diffusion capacity, and 6-min walking distance (6MWD) test. Some underwent lung biopsy.

Rheumatological evaluation. In parallel, all patients underwent an independent rheumatological evaluation (Supplementary Data 2, available with the online version of this article). The assessment included detailed history and physical examination, and complete serological blood tests as mentioned above, with the addition of anti-Jo1, anti-CCP, anti-RNP, anti-Smith, anticentromere, antimyeloperoxidase, antiproteinase 3, and anticardiolipin antibodies, erythrocyte sedimentation rate, various $\mathrm{IgG}$ subclasses including IgG4, and vitamin D level (for the complete list with the normal ranges, see Supplementary Table 1, available with the online version of this article). Additional studies such as antisynthetase antibody were performed as requested by the rheumatologist. In the case of a nonrheumatological ILD, the diagnosis was classified as ILD associated with secondary systemic disorders (Table 2). The rheumatologist was blinded to the MDT decision.

Statistical analysis. All categorical variables are reported as number (\%). They were compared using chi-square analysis and $\mathrm{McNemar}$ test. P values $<0.05$ were considered statistically significant. Sensitivity, specificity, and accuracy were reported. The agreement between the MDT and the rheumatologists was calculated by $\kappa$ statistics. All statistical analyses were performed using SPSS, version 23 software.

Ethical approval. All patients signed an informed consent form. The study was approved by the institutional ethics review committee (No. 0225-12-MMC). The US National Institutes of Health study number is NCT01809574.

\section{RESULTS}

Study population characteristics. Seventy-one patients were screened for the study (Figure 1), but only 60 patients were included. The clinical characteristics of the study population are summarized in Table 1. The study population included 60 patients with the mean age of $67.3 \pm 12$ years, $55 \%$ of whom were male, and $28 \%$ of whom were smokers. While the majority of patients did not have any occupational history (91.6\%), 1 had silica exposure, 1 had asbestos exposure, and 3 had farming-related exposures. The most common clinical findings were dyspnea and Velcro-type crackles (73\% each). Comorbidities included 9 patients with chronic obstructive pulmonary disease, 12 with cardiovascular disorders, and 1 with elevated CRP levels. The pulmonary function tests showed moderate to severe restriction with profound hypoxemia during exercise (87\%). CT findings were ground-glass opacities and honeycombing in $33 \%$ and $38 \%$,

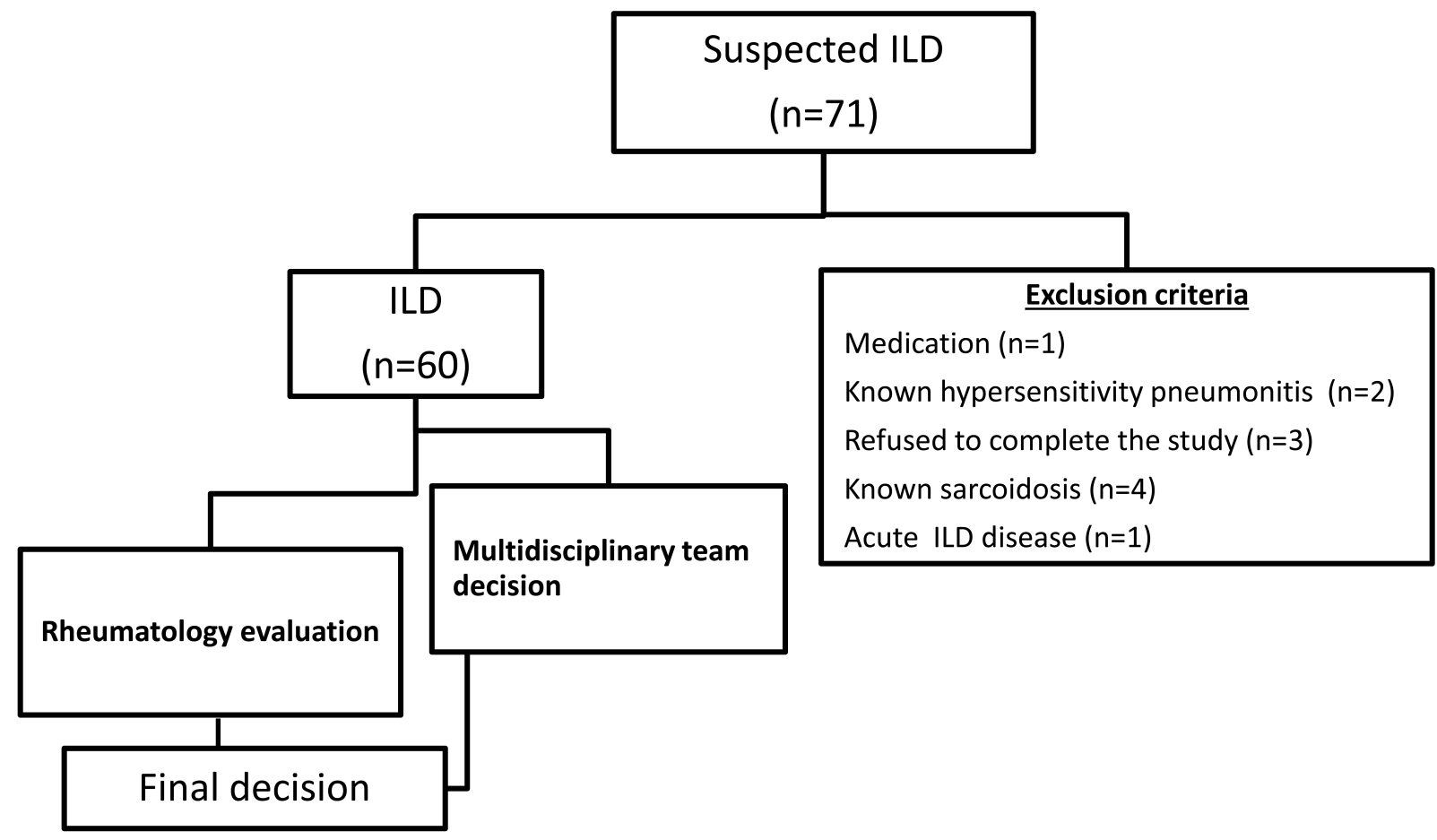

Figure 1. Study design. ILD: interstitial lung disease. 
Table 1. Clinical and demographic characteristics of the study population $(\mathrm{n}=60)$. Values are $\mathrm{n}(\%)$ unless otherwise specified.

\begin{tabular}{|c|c|}
\hline Characteristics & Values \\
\hline \multicolumn{2}{|l|}{ Clinical signs and symptoms } \\
\hline Asymptomatic & $3(5)$ \\
\hline Dyspnea & $44(73)$ \\
\hline Cough & $11(18)$ \\
\hline Chest pain & $1(1.7)$ \\
\hline Velcro crackles & $44(73)$ \\
\hline \multicolumn{2}{|l|}{ Chest radiograph dominant pattern } \\
\hline Reticular pattern & $41(68.3)$ \\
\hline Nodular & $2(3.3)$ \\
\hline Reticulonodular & $8(13.3)$ \\
\hline Infiltrates & $3(5)$ \\
\hline Normal lung parenchyma & $3(5)$ \\
\hline Fibrosis & $3(5)$ \\
\hline \multicolumn{2}{|l|}{ HRCT findings } \\
\hline Reticular pattern & $17(28)$ \\
\hline Honeycombing (UIP pattern) & $23(38)$ \\
\hline Ground-glass opacities & $20(33)$ \\
\hline Bilateral infiltrates & $12(20)$ \\
\hline Mediastinal lymphadenopathy & $13(21)$ \\
\hline \multicolumn{2}{|l|}{ Echocardiogram } \\
\hline Pulmonary hypertension & $28(46)$ \\
\hline Right heart failure & $3(5)$ \\
\hline \multicolumn{2}{|l|}{ Lung function tests, mean $\pm \mathrm{SD}$} \\
\hline $\mathrm{O}_{2}$ saturation & $93.14 \pm 3.75$ \\
\hline DLCO $\%$ & $48 \pm 22$ \\
\hline TLC $\%$ & $66.6 \pm 17.8$ \\
\hline $\mathrm{FVC} \%$ & $72.9 \pm 22.6$ \\
\hline 6-min walking distance, $\mathrm{m}$ & $332.6 \pm 183$ \\
\hline \multicolumn{2}{|l|}{ Bronchoscopy, $\mathrm{n}=23$} \\
\hline BAL only & $9(15)$ \\
\hline TBB & $2(3.4)$ \\
\hline Cryobiopsy & $2(3.4)$ \\
\hline EBUS & $10(16.7)$ \\
\hline
\end{tabular}

HRCT: high-resolution computed tomography; UIP: usual interstitial pneumonia; TLC: total lung capacity; FVC: forced vital capacity; BAL: bronchoalveolar lavage; TBB: transbronchial biopsy; EBUS: endobronchial ultrasound.

respectively, as well as other nonspecific findings of bilateral infiltrates and mediastinal lymphadenopathy. In total, 23 patients (38\%) underwent bronchoscopies (Table 1), while 5 patients had a surgical biopsy. The pathological evaluation of the surgical biopsy results suggested a UIP pattern in 2 cases, nonspecific interstitial pneumonia (NSIP) pattern in 2 cases, and 1 case typical for hypersensitivity pneumonitis (HP).

Rheumatological assessment modified the MDT decision. The diagnosis of patients with ILD according to the MDT, the rheumatologists, and the final diagnosis are shown in Figure 2. The final diagnosis following the rheumatological tests and reevaluation was changed from IPF to alternative, more accurate diagnoses for 6 out of 28 patients (21.4\%). These 6 patients were each diagnosed with a different condition: Sjögren syndrome (SS), antineutrophil cytoplasmic antibodies-associated vasculitis (AAV), antisynthetase syndrome (AS), rheumatoid arthritis (RA), IgG4-related disease
(IgG4-RD), and systemic sclerosis (SSc; Figure 3). Similarly, HP diagnosis was changed from 7 patients to 5 (28.5\%). In general, other diseases related to CTD-ILD with autoimmune features increased following the rheumatological evaluation from 13 to 24 (77\%) on behalf of IPF, NSIP, and HP.

In addition, we found that a routine rheumatological evaluation could have saved 7 bronchoscopies and 1 biopsy. This is because these 8 patients underwent these invasive procedures as part of the MDT routine process, yet eventually were diagnosed with a rheumatologically related condition (Supplementary Table 2, available with the online version of this article). In Table 2, we listed the invasive procedures that could have been prevented by routine rheumatology evaluation $(\mathrm{n}=8)$.

MDT success rates in rheumatology-related ILD diagnosis. An MDT is considered the gold standard for ILD diagnosis ${ }^{5}$. In our study population, 24 of the $60(40 \%)$ patients were eventually diagnosed with a rheumatology-related ILD. To evaluate the significance of the rheumatologist in the final ILD diagnosis, the percentage of "correct" diagnoses by the MDT was calculated. As presented in Figure 3, most cases of SS and RA were successfully diagnosed by the MDT ( $83 \%$ and $75 \%$, respectively). However, the 2 cases of AAV-related ILD were diagnosed only by the rheumatologist, and misdiagnosed as IPF or HP by the MDT. Similarly, only 1 out of 4 cases of AS was diagnosed by the MDT, while the other 3 were misdiagnosed as HP and IPF. IPAF was missed by the MDT in 3 out of 5 cases, and IgG4-RD in 1 of 2 cases. As $40 \%$ of the diagnoses were revised, these results highlight the importance of including rheumatology evaluation as part of the routine ILD diagnosis. In general, the accuracy rate between pulmonologist and rheumatologist was 76.6\% [(34 +12 )/60]. Sensitivity rate was $70.5 \%$ (true positive; $12 / 17$ ) and specificity was $79.1 \%$ (true negative; 34/43). The $\kappa$ measure of agreement between the teams was not high, although it was significant $(0.427, \mathrm{p}<0.001)$.

\section{DISCUSSION}

In our study, we prospectively evaluated the contribution of routine rheumatological assessment to ILD differential diagnosis by comparing the diagnosis before and after the rheumatological evaluation. As predicted, the addition of routine rheumatological evaluation significantly altered the final diagnosis, emphasizing the importance of rheumatologists in the MDT.

In the era of new treatment options for IPF, its exact definition is very important. IPF was the most misdiagnosed condition in our study. This is not surprising, because the diagnosis of IPF is based on the absence of identifiable underlying cause or pathological diagnosis 5 . In our study, 9 IPF cases were eventually diagnosed with a rheumatology-related condition, only because more tests were performed. This is an important finding because the available treatment for IPF and other primary ILD is antifibrotic agents such as

$$
\text { Personal non-commercial use only. The Journal of Rheumatology Copyright } \subset \text { } \text { 2018. All rights reserved. }
$$


A

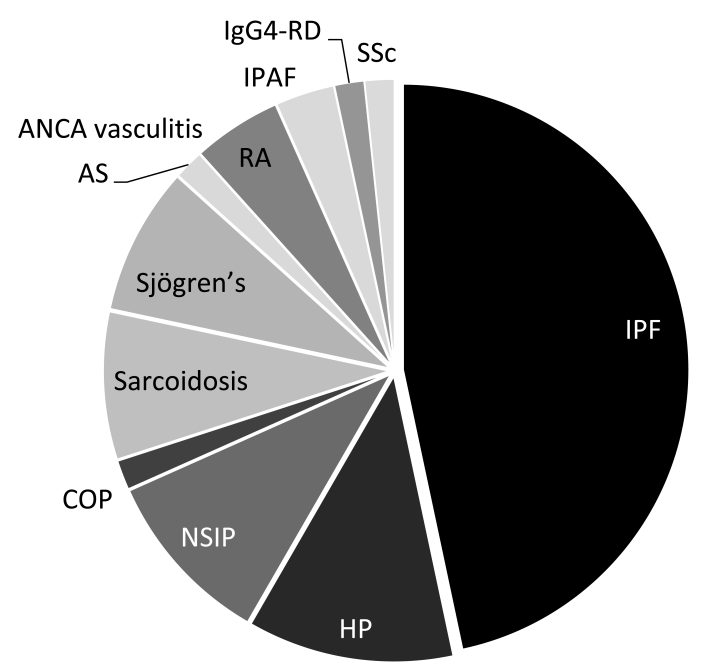

B

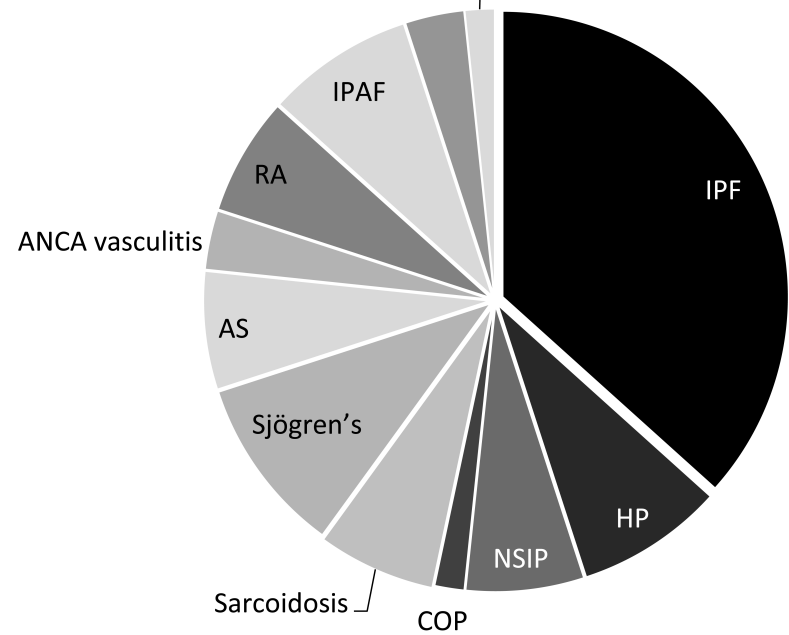

Figure 2. The final diagnosis is altered following rheumatological evaluation. A. The initial multidisciplinary decision. B. The final diagnosis following the rheumatological evaluation. ANCA: antineutrophil cytoplasmic antibody; AS: antisynthetase syndrome; COP: cryptogenic organizing pneumonia; HP: hypersensitivity pneumonitis; IgG4-RD: immunoglobulin G4-related disease; IPAF: interstitial pneumonitis with autoimmune features; IPF: idiopathic pulmonary fibrosis; NSIP: nonspecific interstitial pneumonia; RA: rheumatoid arthritis; SSc: systemic sclerosis.

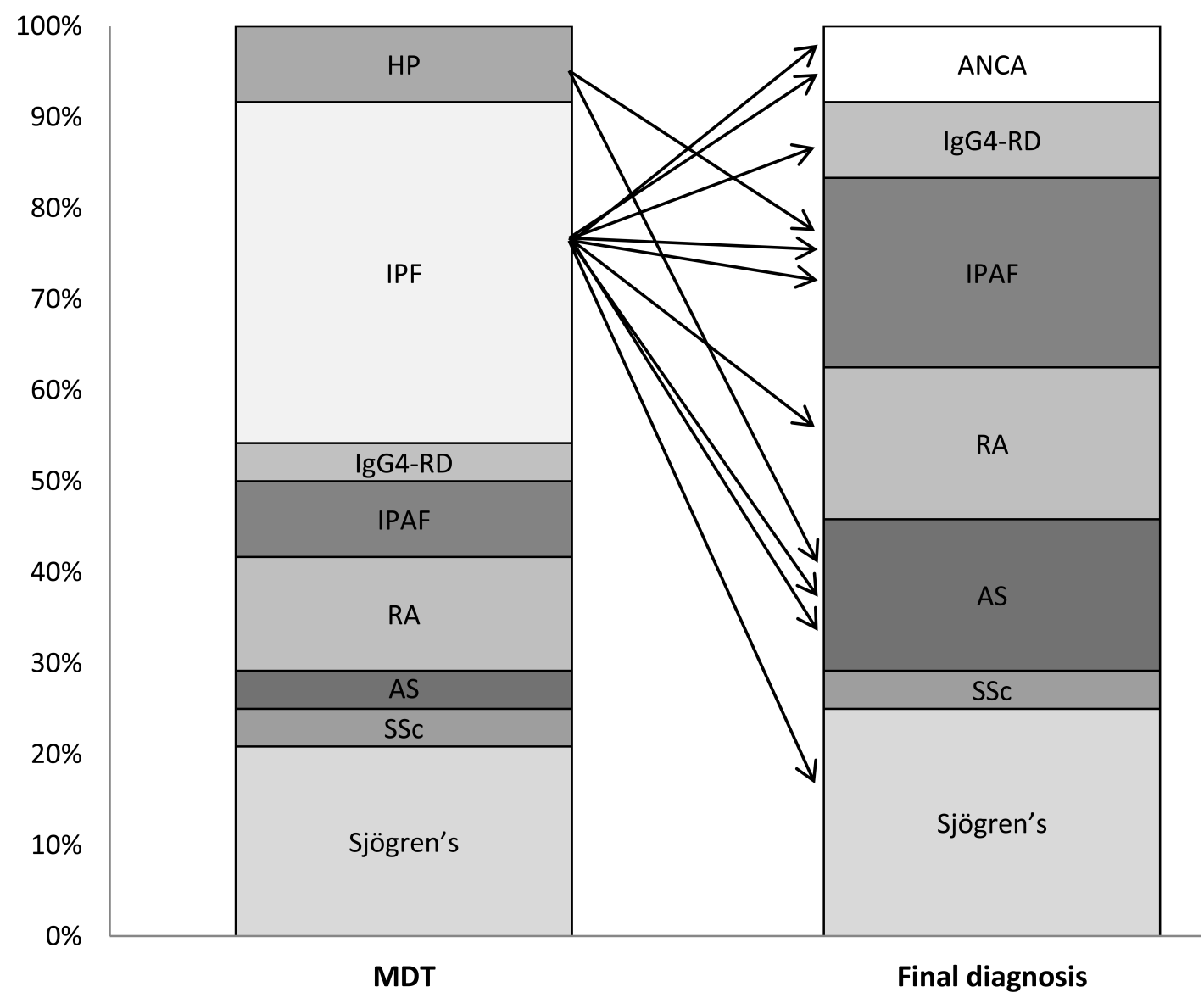

Figure 3. A schematic illustration of changes in diagnosis for patients who were eventually diagnosed with a rheumatologically related condition $(\mathrm{n}=24)$. Arrows indicate the change in diagnosis for each patient $(\mathrm{n}=11)$. ANCA (vasculitis): antineutrophil cytoplasmic antibody vasculitis; AS: antisynthetase syndrome; HP: hypersensitivity pneumonitis; IgG4-RD: immunoglobulin G4-related disease; IPAF: interstitial pneumonitis with autoimmune features; IPF: idiopathic pulmonary fibrosis; MDT: multidisciplinary team; RA: rheumatoid arthritis; SSc: systemic sclerosis. 
Table 2. Invasive procedures that could been prevented by routine rheumatological evaluation $(n=8)$.

\begin{tabular}{lcc}
\hline Pulmonologist's Diagnosis & Final Diagnosis & $\mathrm{N}$ \\
\hline Bronchoscopies, $\mathrm{n}=7$ & & \\
Hypersensitivity pneumonitis & Antisynthetase syndrome & 1 \\
Sarcoidosis & Rheumatoid arthritis & 1 \\
Hypersensitivity pneumonitis & IPAF & 2 \\
IPF & Sjögren syndrome & 2 \\
IPF & SSc & 1 \\
Open lung biopsy & & \\
IPF (possible UIP) & Antisynthetase syndrome & 1 \\
\hline
\end{tabular}

IPF: idiopathic pulmonary fibrosis; IPAF: interstitial pneumonitis with autoimmune features; UIP: usual interstitial pneumonia; SSc: systemic sclerosis.

pirfenidone or nintedanib that are aimed at halting disease progression. On the other hand, ILD that present with autoimmune features are treated with antiinflammatory agents including steroids, cyclophosphamide, mycophenolate mofetil, or rituximab ${ }^{7}$. Therefore, quick and proper diagnoses are crucial for these patients, as misdiagnosis can result in delayed treatment and irreversible lung damage.

The rheumatological assessment included a detailed history and physical examination, and a complete serological blood test set. This integrated approach led to a more precise ILD etiology. Although complete serological assessment alone could improve the diagnosis rate, a detailed history and physical examination by a rheumatologist were very important in the assessment and therefore should be a part of the routine evaluation. Moreover, the serological set that was done in this study was very broad, and perhaps the rheumatologist's evaluation would reach a diagnosis without such a wide range of tests.

In general, association with CTD or other diseases related to ILD provides a context for extrapulmonary disease manifestations, emphasizes the need for surveillance of specific extra thoracic features, and guides management decisions ${ }^{4}$. SS, as well as RA and SSc, were diagnosed by the MDT in about $75 \%$ of the cases without the need for a rheumatological evaluation. However, we noted that several diseases were often missed by pulmonologists and the MDT, and were only diagnosed through a rheumatological evaluation (e.g., AS, AAV-related ILD, and IgG4-RD) ${ }^{8}$.

AS is an autoimmune condition, characterized by antibodies directed against an aminoacyl-tRNA synthetase, along with clinical features that can also include ILD, which is more prevalent and severe in patients with AS, compared to other inflammatory myopathies ${ }^{9,10}$. IgG4-RD is a chronic fibroinflammatory condition characterized by infiltrative fibrosis and lymphoplasmacytic infiltrates, involving 1 or several anatomic sites and often, but not always, with an elevated IgG4 serum level ${ }^{8}$. Awareness of this condition has increased significantly over the last decade, but its cause and pathogenesis are not completely understood ${ }^{8}$. A previous consensus statement suggested using the following major histopathologic features to diagnose IgG4-RD: (1) dense lymphoplasmacytic infiltrate; (2) fibrosis, arranged at least focally in a storiform pattern; and (3) obliterative phlebitis ${ }^{5}$. Compared with other sites, pulmonary involvement of IgG4-RD is often difficult to recognize because of a relative paucity of pathognomonic features and a plethora of overlapping findings with other fibroinflammatory diseases ${ }^{9,10}$. Moreover, storiform fibrosis and obliterative phlebitis can be minimal or absent in the lung ${ }^{8}$. Altogether, CTD-ILD is associated with a more favorable prognosis than IPF of equivalent severity ${ }^{11-16}$. These findings could be related to the histopathology of CTD-ILD, which in most cases is NSIP and less frequently UIP ${ }^{5,6}$. As shown in our study, without the rheumatological evaluation, both AS and IgG4-RD can be missed and misdiagnosed as IPF by the MDT.

This study had several limitations, including the relatively small sample size. In addition, pulmonology evaluations were performed by several physicians. However, the prospective study design, uniform questionnaires, and separate blinded evaluations have increased the accuracy of the results.

Although the guidelines suggest some serological tests when CTD-ILD is suspected, the precise recommendations include only a limited panel. Moreover, not all pulmonologists are familiar with the complete serological profile that rheumatologists often perform in these suspected cases and the detailed history and physical examination related to CTD. Therefore, it is essential to add a rheumatologist to the MDT to conduct a routine rheumatological assessment to increase the diagnostic accuracy of uncommon ILD-related conditions, such as AS, AAV, and IgG4-RD.

\section{ACKNOWLEDGMENT}

The authors thank Tatiana Epstein for the English editing. The authors also thank Faye Schreiber for the editorial support and English editing, and Nava Jelin for the statistical analysis.

\section{ONLINE SUPPLEMENT}

Supplementary material accompanies the online version of this article.

\section{REFERENCES}

1. American Thoracic Society, European Respiratory Society. American Thoracic Society/European Respiratory Society International Multidisciplinary Consensus Classification of the Idiopathic Interstitial Pneumonias. This joint statement of the American Thoracic Society (ATS), and the European Respiratory Society (ERS) was adopted by the ATS board of directors, June 2001 and by the ERS Executive Committee, June 2001. Am J Respir Crit Care Med 2002;165:277-304.

2. Bradley B, Branley HM, Egan JJ, Greaves MS, Hansell DM, Harrison NK, et al; British Thoracic Society Interstitial Lung Disease Guideline Group, British Thoracic Society Standards of Care Committee; Thoracic Society of Australia; New Zealand Thoracic Society; Irish Thoracic Society. Interstitial lung disease guideline: the British Thoracic Society in collaboration with the Thoracic Society of Australia and New Zealand and the Irish Thoracic Society. Thorax 2008;63 Suppl 5:v1-58.

Personal non-commercial use only. The Journal of Rheumatology Copyright $\odot$ 2018. All rights reserved. 
3. Vij R, Strek ME. Diagnosis and treatment of connective tissue disease-associated interstitial lung disease. Chest 2013;143:814-24.

4. Fischer A, du Bois R. Interstitial lung disease in connective tissue disorders. Lancet 2012;380:689-98.

5. Tomassetti S, Piciucchi S, Tantalocco P, Dubini A, Poletti V. The multidisciplinary approach in the diagnosis of idiopathic pulmonary fibrosis: a patient case-based review. Eur Respir Rev 2015;24:69-77.

6. Mathai SC, Danoff SK. Management of interstitial lung disease associated with connective tissue disease. BMJ 2016;352:h6819.

7. Travis WD, Costabel U, Hansell DM, King TE Jr, Lynch DA, Nicholson AG, et al; ATS/ERS Committee on Idiopathic Interstitial Pneumonias. An official American Thoracic Society/European Respiratory Society statement: Update of the international multidisciplinary classification of the idiopathic interstitial pneumonias. Am J Respir Crit Care Med 2013;188:733-48.

8. Schneider F, Veraldi KL, Levesque MC, Colby TV, S Yi E. IgG4-related lung disease associated with usual interstitial pneumonia. Open Rheumatol J 2016;10:33-8.

9. Orlandi M, Barsotti S, Cioffi E, Tenti S, Toscano C, Baldini C, et al. One year in review 2016: idiopathic inflammatory myopathies. Clin Exp Rheumatol 2016;34:966-74.

10. Piroddi IM, Ferraioli G, Barlascini C, Castagneto C, Nicolini A. Severe respiratory failure as a presenting feature of an interstitial lung disease associated with anti-synthetase syndrome (ASS). Respir Investig 2016;54:284-8.
11. Bouros D, Wells AU, Nicholson AG, Colby TV, Polychronopoulos V, Pantelidis P, et al. Histopathologic subsets of fibrosing alveolitis in patients with systemic sclerosis and their relationship to outcome. Am J Respir Crit Care Med 2002;165:1581-6.

12. Park JH, Kim DS, Park IN, Jang SJ, Kitaichi M, Nicholson AG, et al. Prognosis of fibrotic interstitial pneumonia: idiopathic versus collagen vascular disease-related subtypes. Am J Respir Crit Care Med 2007;175:705-11.

13. Fischer A, Swigris JJ, Groshong SD, Cool CD, Sahin H, Lynch DA, et al. Clinically significant interstitial lung disease in limited scleroderma: histopathology, clinical features, and survival. Chest 2008;134:601-5.

14. Lee HK, Kim DS, Yoo B, Seo JB, Rho JY, Colby TV, et al. Histopathologic pattern and clinical features of rheumatoid arthritis-associated interstitial lung disease. Chest 2005; 127:2019-27.

15. Kim DS, Yoo B, Lee JS, Kim EK, Lim CM, Lee SD, et al. The major histopathologic pattern of pulmonary fibrosis in scleroderma is nonspecific interstitial pneumonia. Sarcoidosis Vasc Diffuse Lung Dis 2002;19:121-7.

16. Flaherty KR, Colby TV, Travis WD, Toews GB, Mumford J, Murray $\mathrm{S}$, et al. Fibroblastic foci in usual interstitial pneumonia: idiopathic versus collagen vascular disease. Am J Respir Crit Care Med $2003 ; 167: 1410-5$ 